\title{
La importancia de las unidades de análisis en el modelo de la ecología factorial
}

\author{
Carmen muguruza Cañas * \\ José Miguel Santos Preciado *
}

\section{INTRODUCCIÓN}

La aplicación de la técnica factorial al estudio de la diferenciación residencial de la ciudad, mediante la utilización de variables fundamentalmente demográficas, constituye la denominada Ecología Factorial (Sweester, 1960). Esta técnica de trabajo, muy empleada en los análisis realizados sobre la estructura urbana residencial durante los años sesenta, enlazaba con el planteamiento teórico de Shevky y Bell (Shevky y Bell, 1955), de carácter deductivo, que establecía la existencia en la sociedad moderna de tres ejes fundamentales, capaces de explicar la variación residencial de la ciudad y que podía medirse por tres índices (rango social, urbanización y segregación).

La aplicación de la técnica del análisis factorial al estudio urbano permitía comprobar la validez del modelo de Áreas Sociales de Shevky y Bell, aunque transformando el método de investigación. Se trataba "de caminar hacia el mismo fin, pero evitando la selección de variables a través de una teoría deductiva. En el nuevo método, se obtenían las nuevas variables o factores únicamente sobre la base de la intercorrelación de los datos, utilizando, además, para ello, un extenso número de variables de partida" (Robson, 1969).

El típico estudio de Ecologia Factorial, dentro de la línea anteriormente descrita, ha consistido en la aplicación del análisis factorial a una

* Universidad Nacional de Educación a Distancia 
serie de datos demográficos, socioeconómicos y de vivienda sin ninguna hipótesis de partida, tratando de comprobar, sobre las unidades censales, si los factores que explican la estructura de las variables se ajustan a la diferenciación residencial prevista por los tres ejes clásicos. Precisamente, esta utilización del método factorial, basada en la maximización de la varianza de los datos en el menor número posible de factores, ha condicionado la orientación de los trabajos de investigación primando siempre la coherencia de los resultados estadísticos por encima de la explicación geográfica. La utilización de la técnica factorial de forma atemporal y estática, además de limitar, en gran manera, las posibilidades de este método estadístico, introduce dificultades suplementarias sobre la correcta interpretación de los resultados.

Por este motivo, proponemos un nuevo planteamiento que incorpore tanto la existencia de una hipótesis previa, desde donde dirigir la investigación, como unas unidades de análisis no definidas arbitrariamente, sino seleccionadas en función de los objetivos a conseguir. La principal limitación de la teoría contenida en el Análisis de Áreas Sociales se basa en la ausencia del análisis de los procesos específicamente urbanos, que conducen a la explicación de una realidad determinada. Se trataría por tanto, de establecer una integración "entre el análisis de los procesos sociales y su forma espacial consecuente" (Murdie, 1976). Pensamos, que es difícil conseguir una explicación coherente del modelo de diferenciación residencial de una ciudad o espacio urbano concreto sin hacer una referencia a la intervención en el tiempo de los agentes sociales que modelan la ciudad. Desde esta perspectiva, la elección de unidades espaciales definidas por la homogeneidad en la intervención inmobiliaria 0 en la tipología edificatoria ayudan a deducir los factores claves de la interpretación del modelo de estructura resultante.

Como ejemplo de cuanto venimos señalando, vamos a exponer a continuación el análisis de la diferenciación residencial del municipio de Las Rozas, una de las áreas de suburbanización de alto standing más importante de la periferia metropolitana de Madrid. El estudio está realizado a dos niveles. En primer lugar, utilizando como unidades de análisis las secciones censales municipales $y$, a continuación, unidades que responden a tipologías edificatorias homogéneas. La transparencia en la interpretación de los resultados obtenidos por esta segunda vía de investigación, respecto a la primera, permite establecer la importancia decisiva de las unidades objeto del análisis en la resolución de cualquier problema geográfico planteado. 


\section{LA PROBLEMÁTICA GENERAL DE LAS UNIDADES DE CARÁCTER GEOGRÁFICO}

La delimitación de unas áreas de análisis propias de nuestro estudio viene dada por una reflexión de tipo metodológico sobre el uso de unidades de análisis idóneas para los estudios geográficos.

El problema de la delimitación del espacio en áreas concomitantes, unidades receptáculo de la información geográfica, viene ocupando gran parte de las energías que dedicamos a la metodología de nuestra disciplina. Su importancia se deriva de que "las áreas definidas en un sistema de referencia concreto constituyen las entidades, objetos 0 individuos geográficos, que son en última instancia, las unidades base utilizadas en la medición de los fenómenos espaciales" (Openshaw, 1981).

La problemática específica de la geografía, respecto a otras ciencias sociales, tiene origen en la artificiosidad que caracteriza frecuentemente la delimitación de los elementos base de la investigación. El empleo de unidades espaciales como individuos propios de nuestra disciplina supone la sustitución de los datos de personas u otros atributos geográficos directos, por nuevas entidades cuyas características definitorias son los valores medios de diversos colectivos localizados en los mismos. De esta manera, se atribuyen "a datos de carácter general, conclusiones que corresponden a datos individuales" (Timms, 1976), dando lugar a la denominada falacia ecológica. En este sentido, O. D. Duncam, R. P. Cuzzot y B. Duncam (Duncam, Cuzzot, y Duncam, 1961) diferencian diversos tipos de unidades espaciales, cuyo carácter viene determinado por el tipo de datos que se desean reflejar. Estas unidades pueden ser agrupadas, según estos autores en:

- Áreas consecuencia de una colección de «ítems". Estos “ítems" se refieren a diferentes poblaciones, reflejando alguno de sus atributos (variable cualitativa como sexo, tipo de empleo, etc., o bien directamente cuantificable como renta, etc.). La forma de medir una determinada característica de este tipo puede realizarse bien por medio de un número absoluto o bien como una razón o relación respecto a la población total o superficie.

- Áreas definidas como un segmento del espacio, con una cualidad o un tamaño determinados. Este tipo de unidad espacial serviría para indicar la proporción de superficie diferenciada en varias categorias según el uso o tipo del suelo, vegetación, topografía, etc. 
- Áreas cuyo carácter viene definido respecto al resto de las áreas que componen el territorio completo (como relaciones de flujo existentes entre ellas).

- Áreas donde se desarrollan determinados fenómenos localizados en su interior, que pueden ser reducidos a una o dos dimensiones y asi ser medidas. Podrian ser ríos, carreteras, etc., que a pesar de su carácter tridimensional se reducirían de forma simbólica para facilitar su medición.

Esta posibilidad de construir objetos de análisis geográficos de una manera tan artificiosa nos plantea la duda, para cada estudio concreto, de si habríamos definido correctamente los elementos clave de nuestra investigación. ¿Existe una malla o retícula espacial, que permita extraer los resultados óptimos esperados?

Con excesiva frecuencia, justificada por la dificultad o excesivo costo de la obtención de información, nos hemos acostumbrado al uso de estadísticas contenidas en los censos oficiales, cuyas unidades espaciales han sido definidas, la mayoría de las veces, con criterios puramente administrativos. Aunque estas unidades se refieren a entidades con una personalidad indudable (países, regiones, comarcas, municipios, distritos, secciones censales, etc.), no cabe duda de que su carácter exclusivo las hace inadecuadas a muchos de los estudios realizados.

Frente a esta posición predeterminada existen dos soluciones claramente divergentes. Según la primera, "el sistema de referencia espacial debería ser relativamente independiente del fenómeno que se desea medir” (Batty, 1978). Se trataría de analizar la relación de los fenómenos entre sí independientemente del espacio en que se desarrollan. Desde este punto de vista se nos ofrece la posibilidad de utilizar mallas geométricas (cuadradas, hexagonales, etc.), de unidades regulares de tamaño reducido e idéntica superficie. Este planteamiento tendría la ventaja de permitirnos la integración de estas unidades en áreas de tamaño superior, extraídas estadísticamente, con la finalidad de maximizar algunos de los índices del estudio. Así, «si el análisis se refiere a la estimación del grado de correlación entre dos variables, el procedimiento óptimo para la delimitación de áreas puede incluir la minimización de la covarianza dentro del área y la maximización de la covarianza entre áreas» (Timms, 1976).

Esta posición es por esencia no-geográfica, al olvidar que los sistemas de partición del espacio no pueden ser independientes del fenómeno descrito. Por lo tanto, la otra respuesta sería la de seleccionar las unida- 
des espaciales que mejor se identificaran con el objetivo específico que se desea conseguir.

Siguiendo esta última posición, en esencia geográfica, nosotros intentamos definir una serie de unidades que respondieran a nuestros objetivos de estudio. Como dijimos en la introducción, nuestra intención era ver de que manera se ha ido produciendo la diferenciación social en Las Rozas, y si esta diferenciación tiene una plasmación en el espacio residencial del municipio. Usualmente, los trabajos que pretenden realizar estudios de este tipo vienen utilizando como unidad de análisis las secciones censales, que constituyen la unidad más pequeña que existe en la división administrativa española. Sin embargo, estas unidades normalmente obedecen a un tipo de criterio que en la mayoría de los casos no tiene que ver con los objetivos de los trabajos geográficos, ya que su funcionalidad es otra. Así pues, nosotros quisimos delimitar nuestras propias unidades de investigación en función de los objetivos de la misma. Estos objetivos se basaban en crear la máxima homogeneidad posible en cada unidad de análisis, homogeneidad que viene dada por la existencia de una tipología edificatoria similar, en la creencia que existe una profunda relación entre el tipo de vivienda y las características sociales de la población que en ella reside.

La no utilización de las secciones censales, salvo para la comparación de resultados, no sólo obedece a un criterio de refinamiento del análisis, sino como veremos más adelante a que cada sección censal engloba unidades no homogéneas que hacen que las secciones tengan un grado de heterogeneidad que nos impide llegar a conclusiones válidas. A la hora de rechazar las secciones censales nos llevó a ello un profundo conocimiento del municipio y de la dinámica urbana del mismo. La constatación del acierto de nuestra decisión viene dada por la comparación de los resultados que obtuvimos utilizando las secciones censadas y las unidades por nosotros delimitadas. Resultados que ofreceremos más adelante.

\section{CRITERIO DE DELIMITACIÓN DE LAS ÁREAS DE ANÁLISIS EN EL EJEMPLO UTILIZADO}

Uno de los problemas que nos planteamos era cuál iba a ser el criterio que nos guiara en la definición y delimitación de las áreas de 
análisis. Nosotros optamos por la tipología edificatoria, ya que ésta en el caso de Las Rozas es el resultado del proceso de diferenciación del desarrollo urbano, hecho que queda constatado por las distintas etapas de crecimiento y en cierta forma también son el resultado de la actuación de los distintos tipos de agentes que han intervenido en el proceso de urbanización del municipio. Asi mismo, nos servirá para poner de manifiesto la relación existente entre la composición morfológica de la ciudad medida por la vivienda y el componente social de la misma.

Las tipologías edificatorias las definimos en función de un exhaustivo trabajo de campo y con la ayuda de la fotografía aérea a la hora de estudiar los distintos tipos de trazado.

De esta manera diferenciamos las principales tipologías (Cuadro I), así como su situación geográfica en el municipio (Cuadro II, Figuras 1 y 2).

\section{COMPARACIÓN DE RESULTADOS}

Un punto importante es la comparación entre los resultados obtenidos para las secciones censales y para las áreas definidas por nosotros. Nos hemos centrado en los resultados del primer factor, que no exponemos por limitación de espacio, ya que es el factor más significativo a la hora de hacer explícita la diferenciación social y residencial existente en el municipio de Las Rozas.

La ausencia de criterios definidos para la delimitación de las secciones censales ha producido, en algunos casos, la mezcla dentro de las mismas, de edificaciones bastante alejadas físicamente entre sí o bien de calidad o tipología diferente. Este hecho ha dado lugar a la existencia de unidades espaciales heterogéneas, lo cual resta validez a los resultados obtenidos.

Como se puede observar en el Cuadro III, las secciones censales (Figuras 3 y 4), unidades de mayor tamaño, han promediado los resultados obtenidos para las áreas. La heterogeneidad tipológica de las secciones nos impide apreciar la diferenciación social existente en el municipio según el tipo de vivienda.

En la sección primera, compuesta por viviendas rurales y edificios de calidad mediana y alta, ha primado en su resultado las características 
La importancia de las unidades de análisis en el modelo...

CUADRO I. RESUMEN DE TIPOLOGÍAS

\begin{tabular}{|c|c|c|}
\hline Identificación & Tipo de edificación & Tipo de trazado \\
\hline $1 \mathrm{a}$ & $\begin{array}{l}\text { - Edificios de tres o } \\
\text { cuatro plantas de buena } \\
\text { calidad. }\end{array}$ & $\begin{array}{l}\text { - En «U» con calles } \\
\text { de fondo de saco. }\end{array}$ \\
\hline $1 b$ & $\begin{array}{l}\text { - Edificios de tres a cinco } \\
\text { plantas de inferior } \\
\text { calidad. }\end{array}$ & - Regular. \\
\hline $2 a$ & - Chalets adosados. & - Hileras. \\
\hline $2 b$ & $\begin{array}{l}\text { - Chalets en parcela } \\
\text { pequeña. }\end{array}$ & $\begin{array}{l}\text { - Sinuoso, tipo } \\
\text { ciudad jardín. }\end{array}$ \\
\hline $2 c$ & $\begin{array}{l}\text { - Chalets en parcela } \\
\text { grande. }\end{array}$ & $\begin{array}{l}\text { - Sinuoso, tipo } \\
\text { ciudad jardín. }\end{array}$ \\
\hline $2 d$ & $\begin{array}{l}\text { - Antiguos chalets tipo } \\
\text { sierra. }\end{array}$ & - Irregular. \\
\hline $2 e$ & $\begin{array}{l}\text { - Parcelaciones } \\
\text { periféricas. }\end{array}$ & - Irregular. \\
\hline $3 a$ & - Viviendas rurales. & - Irregular. \\
\hline $3 b$ & $\begin{array}{l}\text { - Viviendas de regiones } \\
\text { devastadas. }\end{array}$ & $\begin{array}{l}\text { - Regular, en } \\
\text { cuadrícula. }\end{array}$ \\
\hline $3 c$ & $\begin{array}{l}\text { - Barrio obrero de la } \\
\text { Renfe, edificios y } \\
\text { casitas bajas. }\end{array}$ & - Regular en línea. \\
\hline
\end{tabular}

Fuente: EP.

socioeconómicas de las áreas de menor nivel socioeconómico, ocultándonos la existencia en esta sección de un área nueva en la que hay un mayor nivel socioeconómico.

La sección segunda ha promediado las características de dos áreas de tipologías diferentes, de edificios de calidad y edificaciones de menor calidad con rasgos socioeconómicos bien distintos.

La sección tercera es la única que presenta un grado de homogeneidad, habiendo pasado su caracterización socioeconómica a un primer lugar dado esta falta de heterogeneidad.

En la sección cuarta, existe también una mezcla de tipologías diferenciadas, habiendo primado las tipologías de mayor nivel socioeconómico y quedando oculta la existencia de un área más deprimida dentro de esta sección. 


\section{CUADRO II. RELACIÓN ENTRE ÁREAS Y TIPOLOGÍAS}

\begin{tabular}{|c|c|c|}
\hline Áreas & & Tipologías \\
\hline Área 1 & & $3 a, 3 b$ \\
\hline Área 2 & & $1 b, 3 a, 2 e$ \\
\hline Área 3 & $\ldots \ldots$ & $1 b$ \\
\hline Área 4 & $\ldots \ldots$ & $2 d$ \\
\hline Área 5 & $\ldots \ldots$ & $1 a$ \\
\hline Área 6 & $\ldots \ldots$ & $1 a$ \\
\hline Área 7 & $\ldots$. & $1 b$ \\
\hline Área 8 & $\ldots \ldots$ & $2 b, 2 d$ \\
\hline Área 9 & $\ldots$ & $1 a, 2 a$ \\
\hline Área 10 & & $2 b$ \\
\hline Área 11 & & $1 b$ \\
\hline Área 12 & $\ldots$ & $2 e$ \\
\hline Área 13 & $\ldots$. & $2 a$ \\
\hline Área 14 & $\ldots \ldots$ & $2 c$ \\
\hline Área 15 & $\ldots$. & $2 b$ \\
\hline Área 16 & & $1 a$ \\
\hline Área 17 & & $2 c$ \\
\hline Área 18 & $\cdots$ & $2 b, 2 c$ \\
\hline Área 19 & $\ldots \ldots$ & $2 c$ \\
\hline Área 20 & $\ldots$. & $2 c$ \\
\hline Área 21 & & $2 b, 2 c$ \\
\hline Área 22 & $\ldots \ldots$ & $2 d$ \\
\hline Área 23 & . & $3 c$ \\
\hline
\end{tabular}

Fuente: EP.

La sección quinta también presenta heterogeneidad de tipologias, mezclándose áreas de alto nivel social con tipologías de nivel inferior, habiendo tenido mayor peso estas últimas.

En la sección sexta, también heterogénea, sin embargo, han primado las características de las tipologias de mayor nivel socioeconómico, ocultándose las áreas más deprimidas que en esta sección existen.

La sección séptima, incluye tipologías de alto nivel socioeconómico, pero de distinto grado, habiéndose realizado un promedio de las mismas.

En la sección octava también existe una cierta mezcla de tipologías, aunque en esta sección, que se corresponde con Las Matas, predomina 
La importancia de las unidades de análisis en el modelo...

CUADRO III. COMPARACIÓN DE LOS RESULTADOS OBTENIDOS

\begin{tabular}{|c|c|c|c|}
\hline $\begin{array}{c}\text { Secciones } \\
\text { Censales }\end{array}$ & Tipologias & $\begin{array}{c}\text { Caracteristicas } \\
\text { (Según análisis con } \\
\text { secciones } \\
\text { censales) }\end{array}$ & $\begin{array}{c}\text { Caracteristicas } \\
\text { (Según análisis con áreas } \\
\text { homogénas) }\end{array}$ \\
\hline 1 & $1 b, 3 a, 1 a$ & Muy baja & Media-alta y muy baja \\
\hline 2 & $1 b, 1 c$ & Media alta & Media-alta y baja \\
\hline 3 & $1 \mathrm{a}$ & Alta & Media-alta \\
\hline 4 & $1 \mathrm{a}, \mathrm{b}, 2 \mathrm{~d}, 2 \mathrm{a}$ & Media-alta & $\begin{array}{c}\text { Alta, media-alta, y } \\
\text { muy baja }\end{array}$ \\
\hline 5 & $\begin{array}{r}1 \mathrm{a}, 3 \mathrm{a}, 2 \mathrm{~d} \\
2 \mathrm{c}, 2 \mathrm{~b}\end{array}$ & Muy baja & Media-alta y muy baja \\
\hline 6 & $2 b, 1 b, 2 a, 2 c$ & Media-alta & $\begin{array}{c}\text { Alta, media-alta, y } \\
\text { muy baja }\end{array}$ \\
\hline 7 & $2 b, 2 c$ & Media-alta & Alta, media-alta \\
\hline 8 & $2 d, 2 b, 2 c$ & Muy baja & $\begin{array}{l}\text { Alta, media-alta y muy } \\
\text { baja }\end{array}$ \\
\hline 9 & $3 c, 2 d, 2 c$ & Media-alta & $\begin{array}{l}\text { Alta, media-alta y muy } \\
\text { baja }\end{array}$ \\
\hline
\end{tabular}

Fuente: EP.

la tipología de antiguos chalets serranos que tienen un nivel socioeconómico muy inferior, que ha prevalecido en su resultado.

Por último, el caso de la sección novena, es un caso clarísimo de mezcla de tipologías marcadamente contrapuestas, por una parte las urbanizaciones de alto standing, por otra el barrio obrero de la Renfe. El menor predominio espacial de las tipologías de bajo status, ha hecho que el promedio sea favorable a las áreas de mayor status, ocultando la existencia en la sección de un área deprimida con marcado carácter proletario.

Comparando los resultados obtenidos entre secciones censales y áreas, creemos que queda claro uno de los objetivos de nuestra investigación que era el poner de manifiesto la necesidad de elaborar unidades significativas a la hora de realizar estudios geográficos.

En los estudios sobre los procesos de diferenciación residencial, pensamos que es la vivienda analizada a través de las distintas tipologías 
edificatorias, la que marca la diferenciación social de la población residente, ya que como ha quedado expuesto, las secciones censales enmarcan unidades tipológicas distintas, dando lugar a resultados promediados que nos impiden apreciar la diferenciación existente en el interior de cada sección censal.

La utilidad de utilizar la vivienda también queda claro a la hora de poder explicar el cómo del proceso de diferenciación social y residencial, ya que las actuaciones de los agentes sociales que han venido interviniendo en este proceso, van dirigidas a la creación de distintos tipos de vivienda en función de la demanda. 
Figura 1.-Áreas de análisis (a). Fuente: EP.

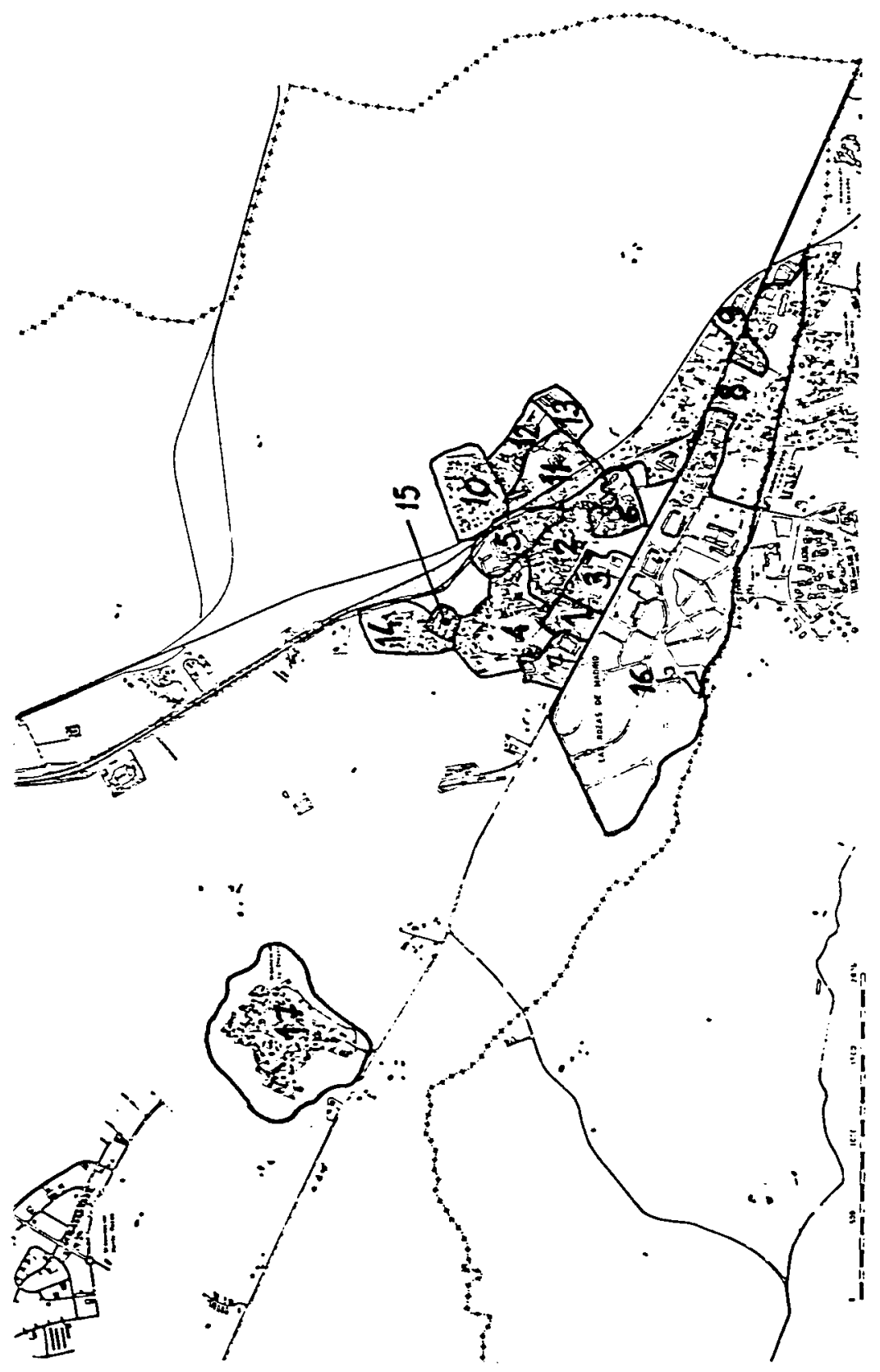


Figura 2.-Áreas de análisis (b). Fuente: EP.

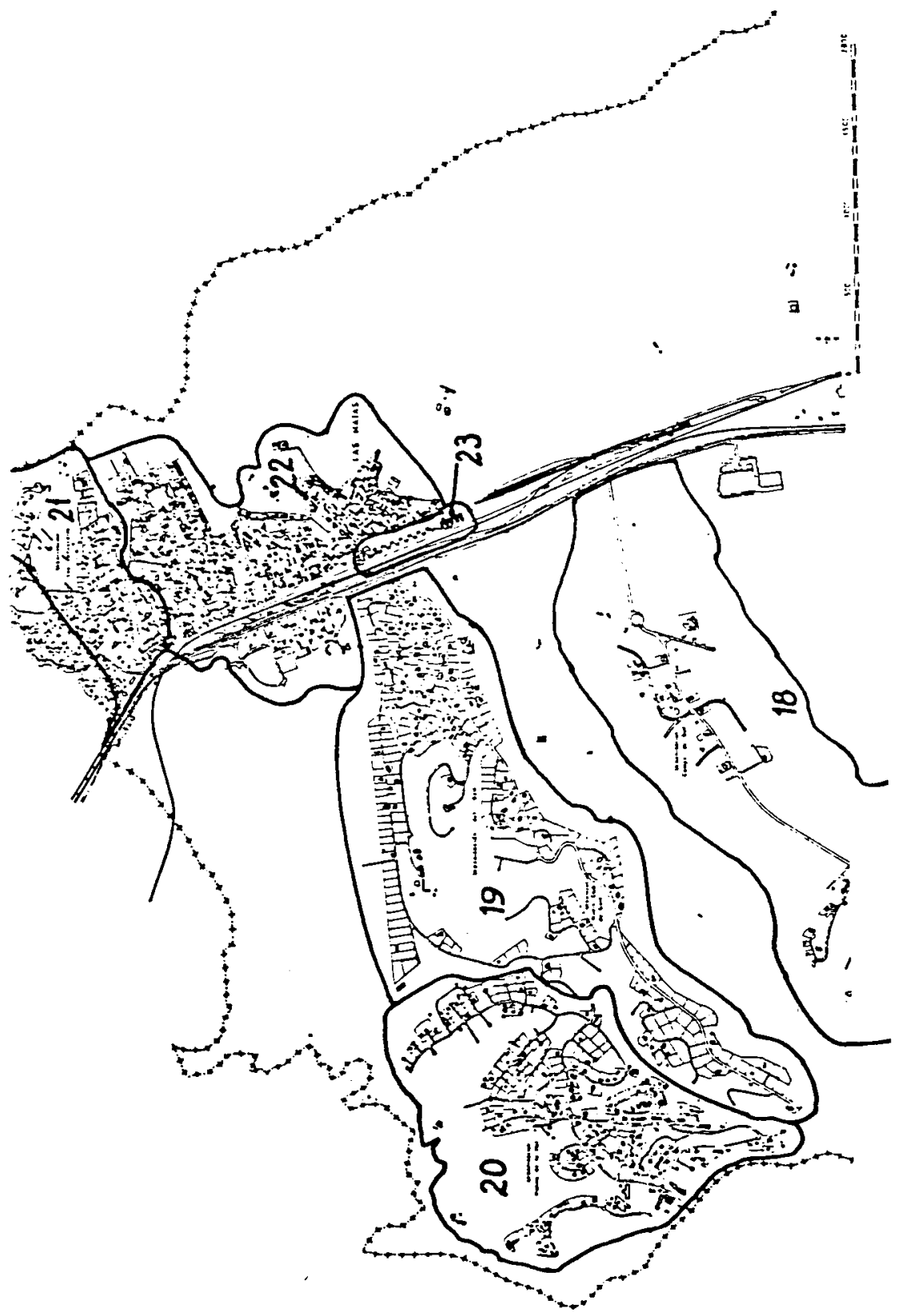


Figura 3.-Secciones Censales (a).

Fuente: EP.

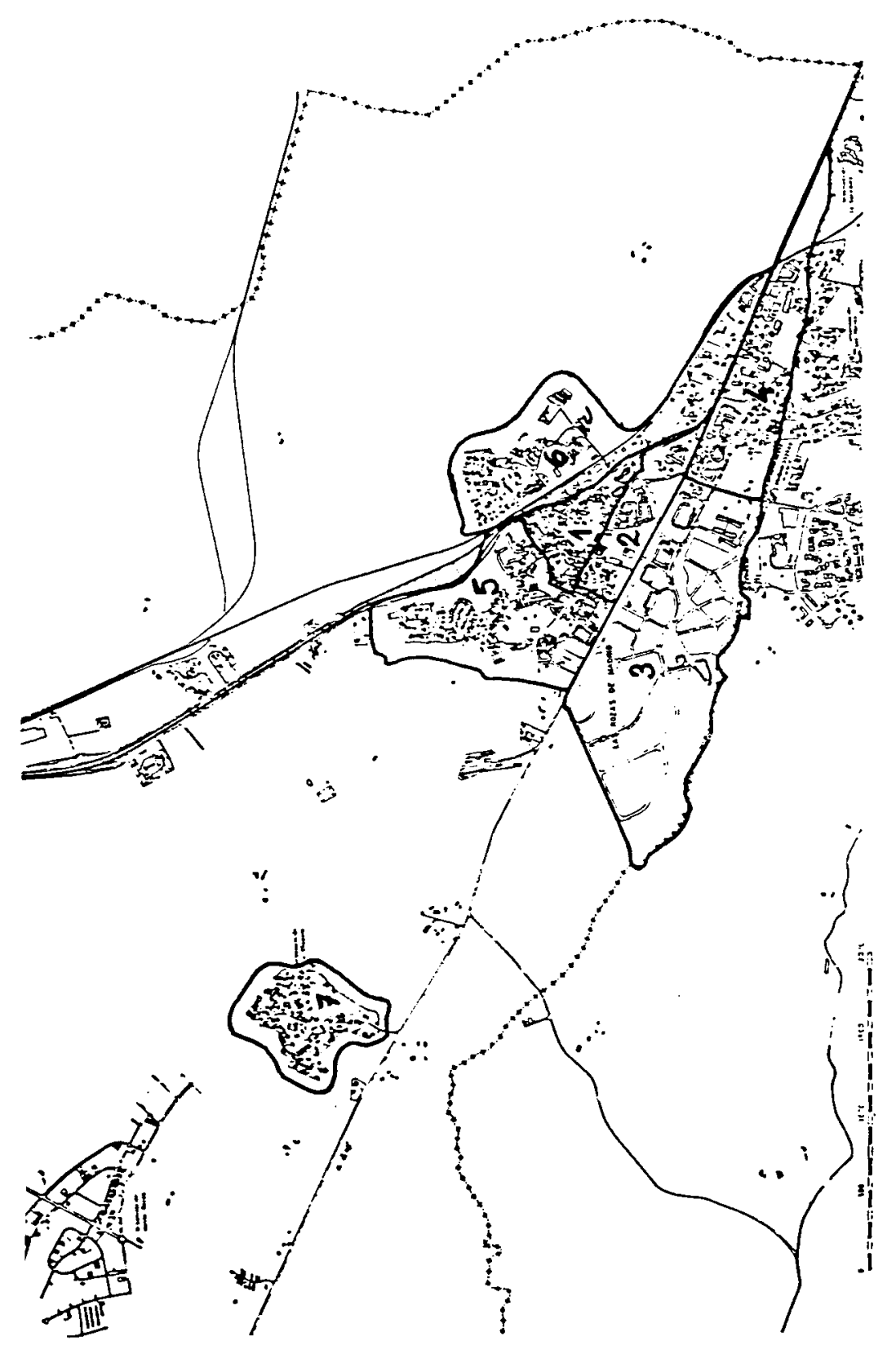


Figura 4.-Secciones Censales (b).

Fuente: EP.

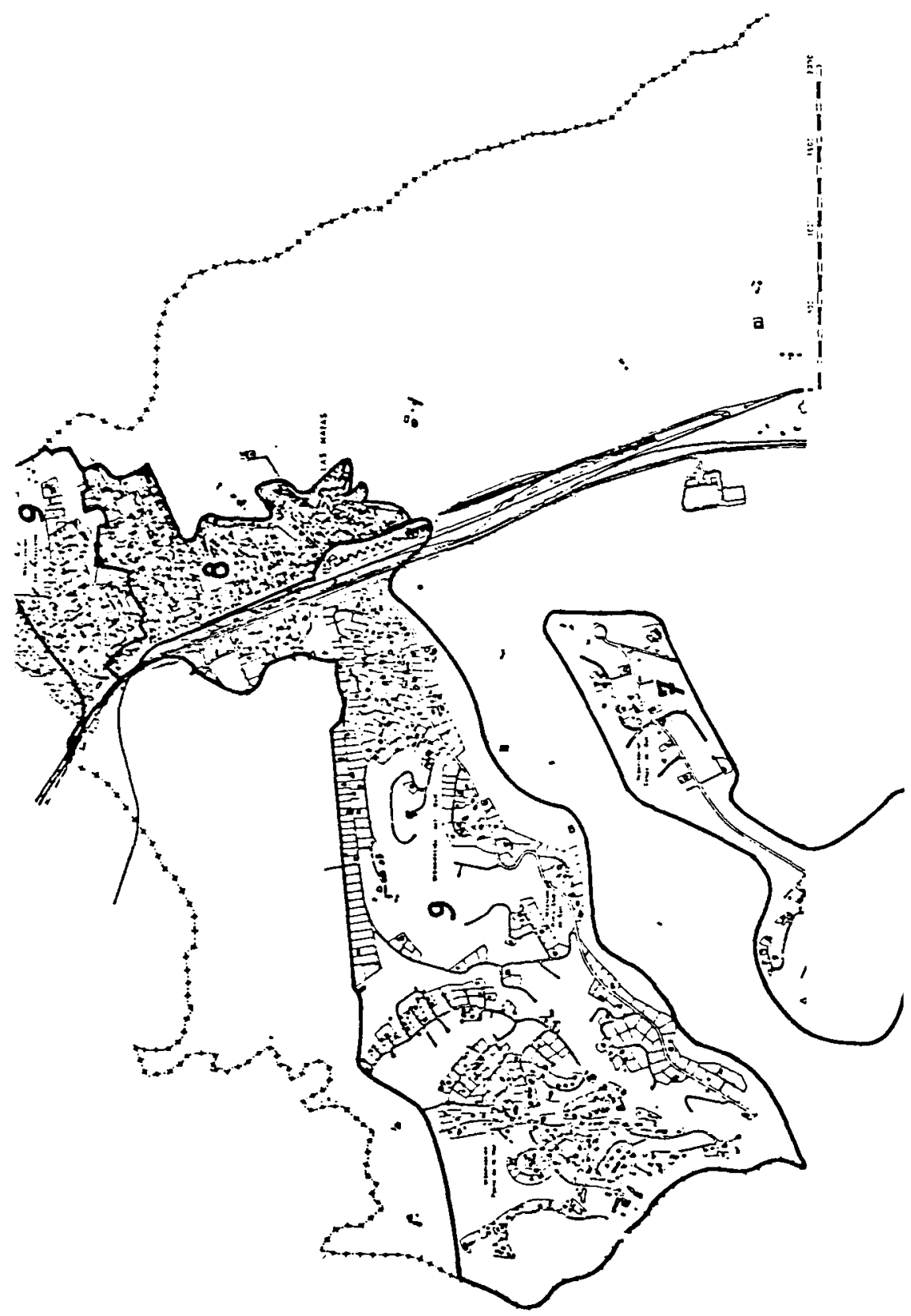




\section{BIBLIOGRAFÍA}

BATTY, M., 1978: "Speculations on a information theorical approach to spatial representation", en Spatial Representation and Spatial Interaction. Leiden, Nighoff, I. Masser y P. Brown (Edit.), p. 146.

Duncam, O. D., Cuzzot, R. P. y Duncam, B., 1961: Statistical Geography. N. York. The Free Press.

OPENSHAW, S., 1981: «Le probleme de l'agregation spatiale en Geographie». L'espace geographique, n..$^{\circ}$. París, p. 15-24.

Murdie, R., 1976: "Spatial Form in the Residential Mosaic», en Herbert, D. y Jhonson, R. J., Spatial Processes and Form. Wiley and Sons.

Robson, B. J., 1969: Urban Analysis: a study of city structure. CUP.

SHEVKY, E. y BELL, W., 1955: Social Area Analysis. Stanford.

SWEESTER, F. L., 1960: Factorial Ecology of Helsinki.

TIMms, D., 1976: El mosaico urbano. Madrid, IEAL. 